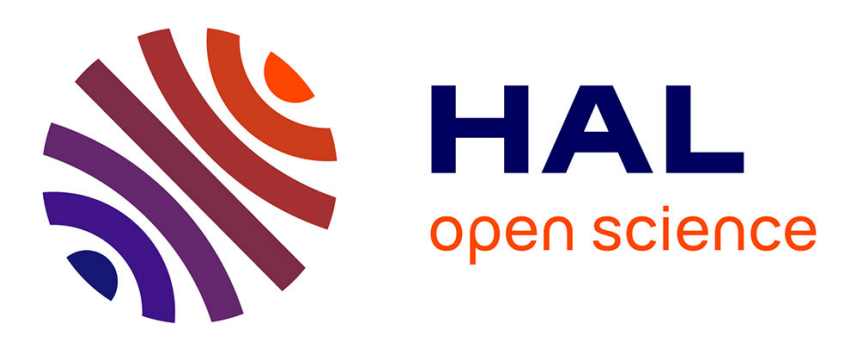

\title{
Caractérisation de l'interface couche implantée substrat semi-isolant LEC GaAs
}

\author{
J.P. David, A. Roizes, M. Bonnet, N. Visentin, J. Icole
}

\section{To cite this version:}

J.P. David, A. Roizes, M. Bonnet, N. Visentin, J. Icole. Caractérisation de l'interface couche implantée substrat semi-isolant LEC GaAs. Revue de Physique Appliquée, 1983, 18 (12), pp.751-756. 10.1051/rphysap:019830018012075100 . jpa-00245142

\section{HAL Id: jpa-00245142 https://hal.science/jpa-00245142}

Submitted on 1 Jan 1983

HAL is a multi-disciplinary open access archive for the deposit and dissemination of scientific research documents, whether they are published or not. The documents may come from teaching and research institutions in France or abroad, or from public or private research centers.
L'archive ouverte pluridisciplinaire HAL, est destinée au dépôt et à la diffusion de documents scientifiques de niveau recherche, publiés ou non, émanant des établissements d'enseignement et de recherche français ou étrangers, des laboratoires publics ou privés. 


\title{
Caractérisation de l'interface couche implantée substrat semi-isolant LEC GaAs
}

\author{
J. P. David, A. Roizes \\ O.N.E.R.A.-C.E.R.T., 2, avenue E. Belin, B.P. 4025, 31055 Toulouse Cedex, France
}

M. Bonnet, N. Visentin

Thomson-CSF LCR, Domaine de Corbeville, 91400 Orsay, France

et J. Icole

Thomson-CSF DCM, Domaine de Corbeville, 91400 Orsay, France

(Reçu le 27 juillet 1983, accepté le 29 août 1983)

\begin{abstract}
Résumé. - Nous avons étudié l'influence des conditions de tirage des lingots semi-isolants GaAs par la méthode LEC sur les pièges présents à l'interface couche implantée substrat semi-isolant. Des expériences de relaxation de pièges à l'interface et d'effet Hall ont été mises en ouvre à cet effet. Le taux d'humidité de l'encapsulant de tirage modifie la compensation côté substrat par un centre accepteur HL 10. Un enrichissement en arsenic de la stoechiométrie initiale du bain de tirage permet l'insertion du fer en site gallium et une inversion plus importante des propriétés électriques du substrat semi-isolant à l'interface. Il existe une corrélation entre les centres de compensation à l'interface et les mobilités moyennes des couches.
\end{abstract}

\begin{abstract}
We have studied the influence of LEC GaAs ingot growth conditions on the deep traps at the interface of implanted layer and semi-insulating substrate. Hall effect and deep trap relaxation experiments have been made. The $\mathrm{B}_{2} \mathrm{O}_{3}$ encapsulant humidity level induced an acceptor $\mathrm{HL} 10$ centre in the substrate after implantation and annealing. An initial arsenic rich stoichiometry of the melt allows iron insertion on gallium sites and a greater substrate effect. There is a correlation between compensation centres and mean mobilities of the implanted layers.
\end{abstract}

\section{Introduction.}

Pour la réalisation de circuits intégrés GaAs, les tensions de pincement des composants élémentaires doivent être contrôlées à $5 \%$ sur l'ensemble du substrat.

Les propriétés individuelles de chacun des transistors à effet de champ sont influencées par les tensions appliquées sur les composants voisins, à travers l'interface couche implantée substrat semi-isolant. Cet effet substrat modifie l'épaisseur électriquement active de la couche implantée, parfois dans de fortes proportions, suivant la densité de centres profonds présents.

La disponibilité de substrats semi-isolants LEC non dopés permet de minimiser la densité de centres profonds de compensation présents à l'interface par rapport aux substrats compensés au chrome.
Cependant, l'implantation ionique induit des défauts dans le réseau GaAs qui modifient sensiblement les propriétés électriques des substrats, après recuit et activation électrique des impuretés implantées.

Dans cette étude, nous avons fait varier les conditions d'élaboration des substrats semi-isolants non dopés, préparés par la méthode LEC et nous avons étudié leur influence sur les centres profonds présents à l'interface couche implantée substrat semi-isolant et leurs effets sur la mobilité moyenne des couches réalisées.

\section{Elaboration des échantillons.}

Cette étude a porté principalement sur l'influence de la nature du creuset (silice ou nitrure de bore), de la qualité de l'oxyde de bore, de la stoechiométrie. 
Avec un creuset en silice, la résistivité des lingots est fortement inhomogène de la tête à la queue et les résultats sont non reproductibles sauf si l'encapsulant présente un taux d'humidité élevé qui empêche l'incorporation de silicium. Avec un creuset en PBN, les lingots sont de résistivité semblable, mais beaucoup plus homogène (Fig. 1).

L'impureté la plus importante est le bore : sa concentration est de l'ordre de $10^{16}$ à $10^{17}$ at. $/ \mathrm{cm}^{3}$.

$\mathrm{La}$ concentration du silicium est inférieure à $10^{15}$ dans le cas du PBN, et de l'ordre de $10^{15}$ en tête du lingot pour les autres échantillons.

La résistivité du matériau varie avec la stochiométrie [1,2] figure 2 .

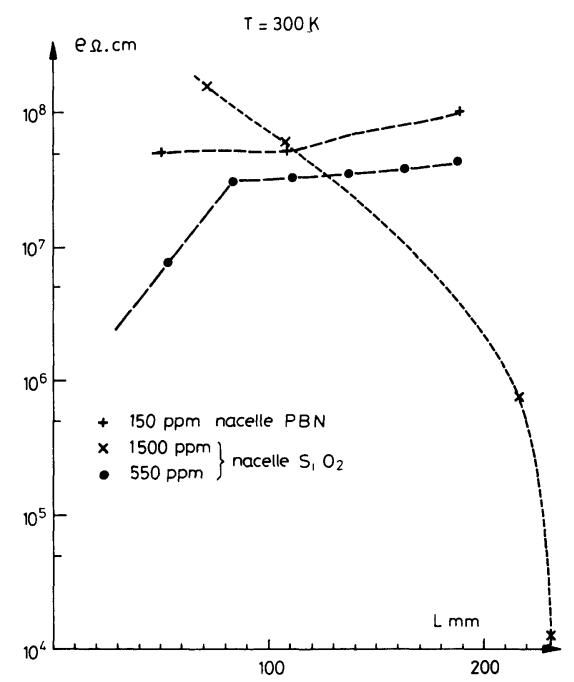

Fig. 1. - Variation typique de résistivité le long de l'axe de tirage avec creuset nitrure et creuset silice.

[Typical resistivity distribution along the ingot for PBN and $\mathrm{SiO}_{2}$ crucibles.]

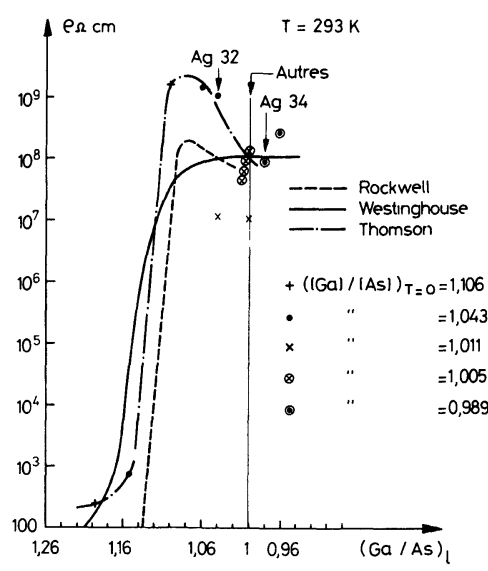

Fig. 2. - Variation de la résistivité des substrats en fonction du taux de stochiométrie initial du bain de tirage.

[Resistivity of the semi-insulating substrate as function of the initial melt stoichiometry.]
Cette variation s'explique par la modification des concentrations d'un niveau donneur profond EL2, d'un niveau accepteur situé à $E_{\mathrm{v}}+77 \mathrm{meV}$ [3].

Dans le cas le plus général, les substrats utilisés ont été tirés avec un rapport initial $\mathrm{Ga} / \mathrm{As}$ du bain proche de 1 , sauf dans le cas des lingots agathe 32 $(\mathrm{Ga} / \mathrm{As}=1,04)$ et agathe $34(\mathrm{Ga} / \mathrm{As}=0,98)$.

Les implantations de silicium, à $150 \mathrm{keV}$ et pour une dose de $3 \times 10^{12}$ at. $/ \mathrm{cm}^{2}$, ont été réalisées à la température ambiante. Les conditions standard de recuit sont $850^{\circ} \mathrm{C}$ pendant $15^{\prime}$. Deux techniques d'encapsulation ont été utilisées, par nitrure et par proximité.

\section{Interface substrat semi-isolant-couche implantée.}

3.1 PRINCIPE DE L'ANALYSE. - Nous nous sommes intéressés à la double zone de charge d'espace à l'interface substrat semi-isolant-couche implantée [4] figure $3 a$.

La charge négative côté substrat est équilibrée par une charge positive côté couche. Les largeurs respectives de ces zones sont liées par la relation $x_{\mathrm{n}} N_{\mathrm{D}}^{+}=x_{\mathrm{p}} N_{\mathrm{A}}^{-}$. Toute variation de charge côté substrat ou côté couche, se traduira par une modulation de l'épaisseur électrique de la couche.

Pour caractériser l'interface, nous avons utilisé la modulation de la conductance de la couche implantée, à la suite d'un changement de la polarisation appliquée au substrat d'une valeur nulle à une valeur négative (Fig. $3 b$ ).

Dans le cas d'un centre côté couche, la diminution de la charge négative excédentaire provoquera une augmentation du courant en fonction du temps, du fait de la diminution de la largeur de la zone de charge d'espace. L'émission de trous côté substrat provoquera une variation inverse sur la couche.

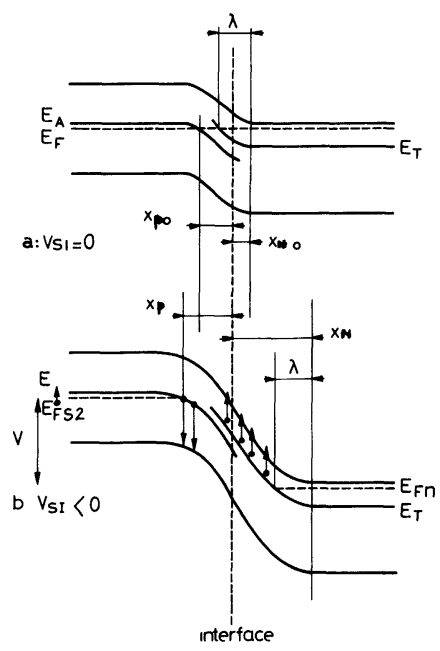

Fig. 3. - Schéma de bande à l'interface couche-substrat : a) $\left.V_{\mathrm{SI}}=0 ; b\right) V_{\mathrm{SI}}<0$.

[Energy band diagram at the layer semi-insulating substrate interface : a) $\left.V_{\mathrm{SI}}=0 ; b\right) V_{\mathrm{SI}}<0$.] 
La densité de centres susceptibles de participer à la relaxation dépend de deux facteurs :

- du remplissage du centre à tension nulle par l'intermédiaire de la densité de porteurs qui est généralement faible dans les zones étudiées,

- de la position du centre par rapport au niveau de Fermi.

3.2 DisPositif EXPÉRIMENTAL. - Nous utilisons, pour la caractérisation, un échantillon d'effet Hall, défini par une technique Mesa, de dimension $30 \times 150 \mu \mathrm{m}$ (Fig. 4).

Les deux contacts extrêmes servent à la mesure du courant, les contacts latéraux à la mesure de la mobilité de Hall.

La tension $V_{\mathrm{DS}}$ utilisée pour l'étude des relaxations de courant est faible, typiquement $0,2 \mathrm{~V}$.

Le contact arrière est assuré par un dépôt de laque d'argent. L'échantillon est placé dans un cryostat sous vide, il peut être éclairé ou à l'obscurité.

Les variations temporelles de courant sont analysées par un voltmètre rapide HP 3437A, piloté par un calculateur HP 9825A.

\section{Résultats obtenus.}

4.1 CARACtÉRISTIQUeS ÉleCtRIQUES DES COUCHES. La variation typique de la conductance des couches en fonction de la température, à tension substrat nulle est indiquée (Fig. 5). Pour comparer les échantillons entre eux, la conductance est normalisée par rapport à la valeur du courant à $80 \mathrm{~K}$.

Les courbes sont très différentes en particulier pour les échantillons d'une même plaquette et montrent des variations parfois importantes dues à la modulation de l'épaisseur électriquement active par les pièges profonds.

Les valeurs de la mobilité moyenne des échantillons étudiés sont indiquées dans le tableau I.

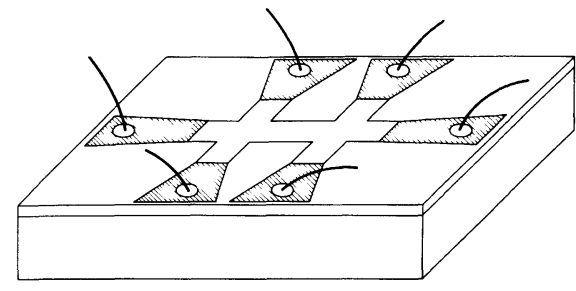

Fig. 4. - Structure de l'échantillon de mesure.

[Sample type used for deep level evaluation and mobility measurement.]

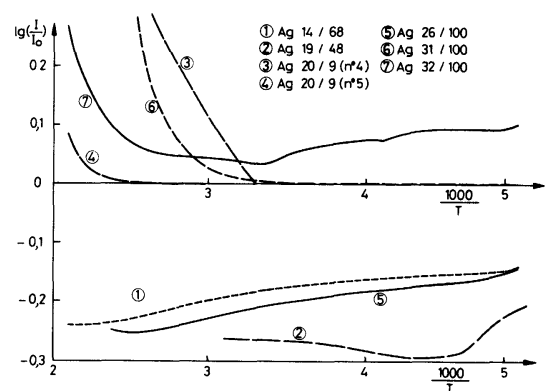

Fig. 5. - Variation relative de conductance d'échantillons de substrats divers, en fonction de $1000 / T$.

[Conductivity evolution as function of temperature for various samples, relative to the low temperature value.]

4.2 CARACtÉRISATION DES CENTRES PROFONDS. - Les centres sont identifiés à partir des abaques de G. M. Martin et al. [5].

Expérimentalement, on observe une dépendance en fonction de la température de l'amplitude des pics DLTS, due au faible nombre de porteurs libres dans les zones étudiées. Les amplitudes mesurées ne sont pas directement liées aux concentrations de chaque centre. L'énergie d'activation de l'amplitude est représentative du niveau de Fermi dans la zone explorée (Fig. 6).

Tableau I. - Conditions de tirage particulières et mobilités moyennes des couches

[Growth conditions and mean layers mobility]

Creuset $\mathrm{SiO}_{2}$

Creuset PBN

\begin{tabular}{|l|c|l|c||l|c|l|c|}
\hline Référence & $\begin{array}{c}{\left[\mathrm{H}_{2} \mathrm{O}\right]} \\
\text { en ppm }\end{array}$ & Encapsulant & $\mu_{\mathrm{H}} \mathrm{cm}^{2} / \mathrm{V} . \mathrm{s}$ & Référence & $\begin{array}{c}{\left[\mathrm{H}_{2} \mathrm{O}\right]} \\
\text { en ppm }\end{array}$ & Encapsulant & $\mu_{\mathrm{H}} \mathrm{cm}^{2} / \mathrm{V} . \mathrm{s}$ \\
\hline Ag. 19/48 & 350 & Proximité & 3700 & Ag. 14/11 & 150 & Proximité & 4200 \\
Ag. 20/9 & 550 & - & 3800 & Ag. 14/68 & 150 & Nitrure CVD & 4300 \\
Ag. 22/174 & 1500 & Nitrure CVD & 5150 & Ag. 23/22(A) & 500 & Proximité & 3200 \\
Ag. 22/193 & 1500 & Proximité & 3950 & Ag. 23/22(B) & 500 & Proximité & 5000 \\
& & & & Ag. 23/112 & 500 & - & 5200 \\
& & & & Ag. 26/100 & 960 & Nitrure pulv. & 5500 \\
& & & & Ag. 31/100 & 520 & Nitrure CVD & 4850 \\
& & & & Ag. 32/100 & 150 & Nitrure pulv. & 4200 \\
& & & & Ag. 34/111 & 150 & - & 3600 \\
\hline
\end{tabular}


Tableau II a. - Amplitude des centres émetteurs de trous (unité arbitraire)

[Hole traps magnitude (arbitrary units)]

\begin{tabular}{|c|c|c|c|c|c|c|c|}
\hline Réf. éch. & $\begin{array}{l}\text { Conditions } \\
\text { particulières } \\
\text { de tirages }\end{array}$ & $\mathrm{HL}_{1}$ & $\mathrm{HL}_{2}$ & $\mathrm{HL}_{3}$ & $\mathrm{HL}_{4}$ & $\mathrm{HL}_{5}$ & $\mathrm{HL}_{10}$ \\
\hline Ag. $19 / 48$ & $\begin{array}{l}350 \mathrm{ppm} \\
\text { Proximité }\end{array}$ & 3 & & 0,3 & $\begin{array}{c}0,7 \text { à } \\
15\end{array}$ & & $\begin{array}{c}0,8 \text { à } \\
300\end{array}$ \\
\hline Ag. $20 / 9$ & $\begin{array}{l}550 \mathrm{ppm} \\
\text { Proximité }\end{array}$ & $\begin{array}{l}15 \text { à } \\
150\end{array}$ & 4 & 10 & 1 & & $\begin{array}{l}20 \text { à } \\
350\end{array}$ \\
\hline Ag. $22 / 174$ & $\begin{array}{l}\text { Nitrure } \\
1500 \text { ppm }\end{array}$ & & $\begin{array}{l}40 \text { à } \\
120\end{array}$ & 70 & & & 2 \\
\hline Ag. $22 / 193$ & $\begin{array}{l}1500 \mathrm{ppm} \\
\text { Proximité }\end{array}$ & & 5 & & & & $\begin{array}{l}1 \mathrm{à} \\
100\end{array}$ \\
\hline Ag. $23 / 22(\mathrm{~A})$ & $\begin{array}{l}500 \text { ppm } \\
\text { Proximité }\end{array}$ & $\begin{array}{l}3 \text { à } \\
40\end{array}$ & $\begin{array}{l}3 \text { à } \\
300\end{array}$ & $\begin{array}{l}2 \mathrm{à} \\
15\end{array}$ & $\begin{array}{l}4 \text { à } \\
100\end{array}$ & & $\begin{array}{c}100 \text { à } \\
400\end{array}$ \\
\hline Ag. $23 / 22$ (B) & $\begin{array}{l}500 \mathrm{ppm} \\
\text { Proximité }\end{array}$ & $\begin{array}{l}20 \text { à } \\
200\end{array}$ & $\begin{array}{c}50 \text { à } \\
500\end{array}$ & 150 & $\begin{array}{l}25 \mathrm{à} \\
200\end{array}$ & & 50 \\
\hline Ag. $23 / 112$ & $\begin{array}{l}500 \mathrm{ppm} \\
\text { Proximité }\end{array}$ & & 40 & $\begin{array}{l}1 \mathrm{à} \\
10\end{array}$ & $\begin{array}{c}0,6 \text { à } \\
10\end{array}$ & & 5 \\
\hline Ag. $26 / 100$ & $\begin{array}{l}\text { Nitrure } \\
960 \text { ppm }\end{array}$ & & 10 & $\begin{array}{l}1 \text { à } \\
30\end{array}$ & & & 20 \\
\hline Ag. $31 / 100$ & $\begin{array}{l}\text { Nitrure } \\
520 \mathrm{ppm}\end{array}$ & & $\begin{array}{l}2 \grave{a} \\
70\end{array}$ & 60 & & & \\
\hline Ag. $32 / 100$ & $\begin{array}{c}\text { Nitrure } \\
150 \mathrm{ppm} \\
(\mathrm{Ga}) /(\mathrm{As})=1,04\end{array}$ & 20 & 40 & 30 & & & \\
\hline Ag. $34 / 111$ & $\begin{array}{c}\text { Nitrure } \\
150 \mathrm{ppm} \\
(\mathrm{Ga}) /(\mathrm{As})=0,98\end{array}$ & & & $\begin{array}{l}500 \text { à } \\
3000\end{array}$ & 4000 & 50 & \\
\hline Ag. $14 / 11$ & $\begin{array}{l}150 \mathrm{ppm} \\
\text { Proximité }\end{array}$ & & & 2 & 7 & & \\
\hline Ag. $14 / 68$ & $\begin{array}{l}\text { Nitrure } \\
150 \mathrm{ppm}\end{array}$ & & 50 & $\begin{array}{l}2 \text { à } \\
40\end{array}$ & & & \\
\hline
\end{tabular}


Tableau II b. - Amplitude des centres émetteurs d'électrons (unité arbitraire).

[Electron traps magnitude (arbitrary units)]

\begin{tabular}{|c|c|c|c|c|c|c|c|c|c|}
\hline Réf. éch. & $\begin{array}{l}\text { Conditions } \\
\text { particulières } \\
\text { de tirages }\end{array}$ & $\mathrm{EL}_{1}$ & $\mathrm{EL}_{2}$ & $\mathrm{EL}_{4}$ & $\mathrm{EL}_{5}$ & $\mathrm{EL}_{6}$ & $\mathrm{EI}_{1}$ & $\mathrm{~EB}_{3}$ & $\mathrm{~EB}_{4}$ \\
\hline Ag. $19 / 48$ & $\begin{array}{l}350 \mathrm{ppm} \\
\text { Proximité }\end{array}$ & & 0,6 & 3 & & & 0,5 & 4 & \\
\hline Ag. 20/9 & $\begin{array}{l}550 \text { ppm } \\
\text { Proxinhité }\end{array}$ & & & & & & 1 & & \\
\hline Ag. $22 / 174$ & $\begin{array}{l}\text { Nitrure } \\
1500 \mathrm{ppm}\end{array}$ & & & & & & & & \\
\hline Ag. $22 / 193$ & $\begin{array}{l}1500 \mathrm{ppm} \\
\text { Proximité }\end{array}$ & & 0,9 & 0,7 & & & 2 & & 1 \\
\hline Ag. $23 / 22$ (A) & $\begin{array}{l}500 \text { ppm } \\
\text { Proximité }\end{array}$ & & $\begin{array}{l}2 \mathrm{à} \\
20\end{array}$ & & & & & 20 & 2 \\
\hline Ag. 23/22 (B) & $\begin{array}{l}500 \mathrm{ppm} \\
\text { Proximité }\end{array}$ & & 40 & 100 & & & & 40 & 50 \\
\hline Ag. $23 / 112$ & $\begin{array}{l}500 \mathrm{ppm} \\
\text { Proximité }\end{array}$ & & 1 & & & & & & $\begin{array}{l}3 \text { à } \\
15\end{array}$ \\
\hline Ag. $26 / 100$ & $\begin{array}{l}\text { Nitrure } \\
960 \mathrm{ppm}\end{array}$ & & & & & & & & 40 \\
\hline Ag. $31 / 100$ & $\begin{array}{l}\text { Nitrure } \\
520 \mathrm{ppm}\end{array}$ & & $\begin{array}{c}0,6 \text { à } \\
10\end{array}$ & & & & & & \\
\hline Ag. $32 / 100$ & $\begin{array}{c}\text { Nitrure } \\
150 \mathrm{ppm} \\
(\mathrm{Ga}) /(\mathrm{As})=1,04\end{array}$ & 8 & 9 & & & & & 20 & \\
\hline Ag. $34 / 111$ & $\begin{array}{c}\text { Nitrure } \\
150 \mathrm{ppm} \\
(\mathrm{Ga}) /(\mathrm{As})=0,98\end{array}$ & 200 & 200 & 1500 & 300 & & & 500 & 200 \\
\hline Ag. $14 / 11$ & $\begin{array}{l}150 \mathrm{ppm} \\
\text { Proximité }\end{array}$ & & & & 7 & 4 & 1 & $\begin{array}{l}1 \mathrm{à} \\
40\end{array}$ & 20 \\
\hline Ag. $14 / 68$ & $\begin{array}{l}\text { Nitrure } \\
150 \mathrm{ppm}\end{array}$ & & 2 & 5 & & & & & \\
\hline
\end{tabular}




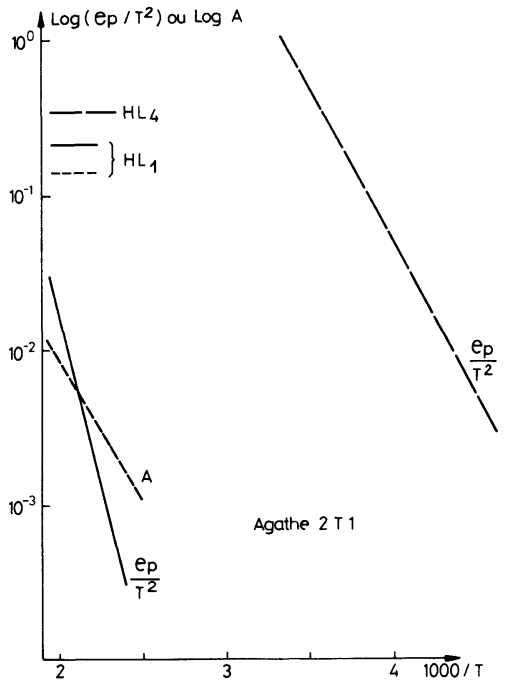

Fig. 6. - Amplitude et coefficient d'émission du centre HL 1 en fonction de la température et coefficient d'émission HL 4 d'un échantillon agathe $2 \mathrm{~T}_{1}$ dopé chrome.

[Amplitude and emission rate of HL 1 centre as function of temperature, compared to HL 4 emission rate for agathe 2 $T_{1}$ chromium doped sample.]

Les résultats obtenus sont consignés dans les tableaux II, respectivement pour les émetteurs d'électrons et de trous.

Qualitativement, les centres profonds assurant la compensation côté substrat semi-isolant sont les mêmes quel que soit le type de creuset utilisé (PBN ou silice) et il existe toujours une zone $\mathrm{P}^{-}$du côté substrat.

L'utilisation d'un taux d'humidité élevé de l'encapsulant de tirage $\mathrm{B}_{2} \mathrm{O}_{3}$, favorise la formation d'un centre accepteur HL 10. L'amplitude associée à ce centre est souvent plus importante que celle de HL 2 dans le cas d'un recuit d'implantation par proximité, mais des fluctuations importantes existent sur une même plaque, par exemple agathe 23/22.

Dans le cas d'une encapsulation nitrure, les amplitudes de ces deux centres sont proches. De plus, l'encapsulation nitrure favorise la création de HL 3.

L'utilisation d'une stochiométrie riche en arsenic (agathe 34) provoque une conductivité fortement $P$ côté substrat. Les amplitudes des centres HL 3, HL 4 et HL 5 sont très importantes comparées aux autres échantillons (effet substrat très important). Il existe de même un effet considérable sur les centres émetteurs d'électrons, côté couche.

Des centres HL 1 sont observés sans aucune corrélation avec la technologie de tirage.

Côté couche, l'amplitude associée à EL 2 est plus importante lors d'une encapsulation nitrure.

4. 3 LES MOBILITÉs. - Des fluctuations importantes des mobilités mesurées sont observées (tableau I). Les mobilités faibles, au plus égales à $4000 \mathrm{~cm}^{2} / \mathrm{V}$.s sont caractéristiques d'une forte compensation, et les causes en sont probablement multiples.

Cependant, on peut remarquer que les échantillons ayant une mobilité faible, sont généralement ceux pour lesquels, un centre accepteur HL 10 et HL 3 est prédominant.

\section{Conclusion.}

Les centres contrôlant la compensation après implantation dans le semi-isolant sont de même nature dans les deux types de creuset. Les différences proviennent des modifications du taux d'humidité, de la stoechiométrie et de la méthode d'encapsulation. Un taux d'humidité élevé de l'encapsulant favorise la formation d'un centre accepteur HL 10. L'encapsulation nitrure favorise la présence de HL 3.

La modification du taux de stœechiométrie vers un enrichissement en arsenic, modifie fortement la compensation.

\section{Bibliographie}

[1] Hobgood, H., Ta, L., Rohatgi, A., Heldridge, G., THOMAs, R., Semi-insulating III-V Materials conference, Evian 1982, p. 28.

[2] Ta, L., Hobgood, H., Rohatgi, A., Thomas, R., Japan. Appl. Phys. 53 (1982) 5771.

[3] Holmes, D., Elliot, K., Chen, R., Kirkpatrick, C., Semi-insulating III-V Materials Conference, Evian 1982 , p. 19.
[4] Rossel, P., Tranduc, H., Graffeuil, J., Azizi, C., Revue Phys. Appl. 12 (1977) 1679.

Rossel, P., Nuzillat, G., Tranduc, H., Bert, G., Graffeuil, J., Azizi, C., Revue Phys. Appl. 13 (1978) 503.

[5] Mitonneau, A., Martin, G. M., Mircea, A., Electron. Lett. 13 (1977) 666.

Martin, G. M., Mitonneau, A., Mircea, A., Electron. Lett. 13 (1977) 191. 